\title{
Tangible Views for Information Visualization
}

\author{
Martin Spindler ${ }^{1}$, Christian Tominski ${ }^{2}$, Heidrun Schumann ${ }^{2}$, Raimund Dachselt ${ }^{1}$ \\ ${ }^{1}$ User Interface \& Software Engineering Group \\ Otto-von-Guericke-University Magdeburg, Germany \\ ${ }^{2}$ Computer Graphics Group \\ University of Rostock, Germany
}

spindler@ovgu.de,ct@informatik.uni-rostock.de, schumann@informatik.uni-rostock.de, dachselt@ovgu.de

\begin{abstract}
In information visualization, interaction is commonly carried out by using traditional input devices, and visual feedback is usually given on desktop displays. By contrast, recent advances in interactive surface technology suggest combining interaction and display functionality in a single device for a more direct interaction. With our work, we contribute to the seamless integration of interaction and display devices and introduce new ways of visualizing and directly interacting with information. Rather than restricting the interaction to the display surface alone, we explicitly use the physical three-dimensional space above it for natural interaction with multiple displays. For this purpose, we introduce tangible views as spatially aware lightweight displays that can be interacted with by moving them through the physical space on or above a tabletop display's surface. Tracking the 3D movement of tangible views allows us to control various parameters of a visualization with more degrees of freedom. Tangible views also facilitate making multiple - previously virtual - views physically "graspable". In this paper, we introduce a number of interaction and visualization patterns for tangible views that constitute the vocabulary for performing a variety of common visualization tasks. Several implemented case studies demonstrate the usefulness of tangible views for widely used information visualization approaches and suggest the high potential of this novel approach to support interaction with complex visualizations.
\end{abstract}

ACM Classification: H5.2 [Information interfaces and presentation]: User Interfaces. - Graphical user interfaces.

\section{General terms: Design, Human Factors.}

Keywords: Tangible views, interaction techniques, magic lenses, tabletop displays, multiple views, focus + context techniques, multi-surface user interfaces.

\section{INTRODUCTION}

In visualization science, it is commonly known that encoding all information in a single image is hardly possible once a data set exceeds certain size or complexity, or when multiple users have to look at the data from different perspectives. This problem can be resolved spatially by providing multiple

Permission to make digital or hard copies of all or part of this work for personal or classroom use is granted without fee provided that copies are not made or distributed for profit or commercial advantage and that copies bear this notice and the full citation on the first page. To copy otherwise, to republish, to post on servers or to redistribute to lists, requires prior specific permission and/or a fee.

ITS'10, November 7-10, 2010, Saarbrücken, Germany.

Copyright 2010 ACM 978-1-60558-745-5/09/10...\$10.00. views on the data [3] or by embedding additional local views in the visualization [5]. It can also be resolved temporally by changing representations over time. Except for a few automatic methods, in most cases changing a visualization is a result of user interaction [46].

Mouse and keyboard are the predominant interaction devices to adjust the representation according to the data and the task at hand. Compared to the richness of available visualization methods, the number of dedicated interaction techniques for information visualization is moderate. Reasons might be that complex interactivity must be squeezed through the degrees of freedom offered by mouse and keyboard and that display and interaction device are physically separated. Recent research on tabletop displays demonstrates that the integration of display and interaction device is beneficial for interactive visualization $[17,18]$. In particular multi-touch gestures strive for naturalness. However, interaction is still mainly based on 2D positional input generated by pointing or moving fingers on the display's surface.

On the other hand, visualizations printed on paper are limited in terms of interactively altering the graphics. However, it is quite intuitive to grab a piece of paper, to move it towards the eyes to see more detail, and to put it back for an overview. Similarly, it is quite easy to fold pages in a report or to arrange multiple printouts on a desk to compare figures side-by-side. Doing so in multiple view environments on a computer display might involve several steps of reconfiguration of the visualization, which may turn out to be cumbersome when using mouse and keyboard alone. In a sense, an advantage of hardcopy visualizations is that they serve as a device for direct interaction and as a display at the same time.

In our research, we aim to narrow the gap between common interaction performed on the display and the natural manipulation we perform with paper. To that end, we developed what we call tangible views. A tangible view is a physical surface, for example a piece of cardboard, that users can hold in their hands. As long as it is handy, there is no restriction on a tangible view's shape and size. A tangible view serves two purposes - it is used as a local display in conjunction with a tabletop display, and it is used as an input device. Display functionality is realized by projecting specific graphical information onto tangible views. The three-dimensional manipulation of a tangible view is tracked in space to make more degrees of freedom available for interacting with the visualization and the data. While a single tangible view can already be a promising alternative to classic interaction, the true potential of our approach lies in the possibility to use multiple tangible views at the same time. In the latter case, tangible 


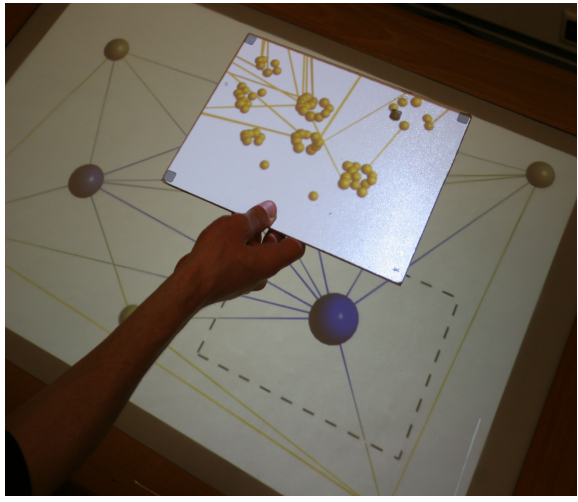

(a) A tangible view is used for smoothly exploring a graph at different levels of abstraction.

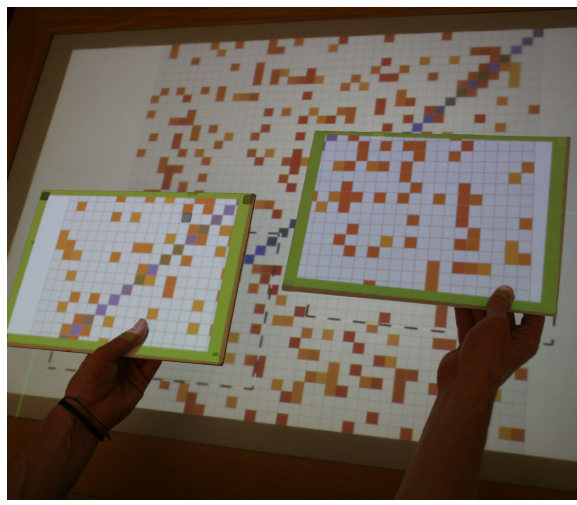

(b) Using multiple tangible views simultaneously facilitates visual comparison tasks.

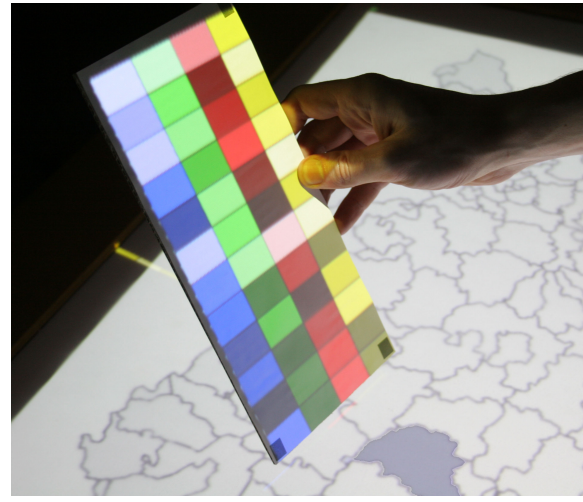

(c) Tangible views can be used to augment a map display with additional visual representations.

Figure 1: Tangible views as spatially-aware, handheld displays introduce new ways of visualizing and interacting with information.

views do not only cater for natural interaction, but they also supersede virtual multiple views by physical ones, which can be freely moved in space. In summary, tangible views:

1. Integrate display and interaction device. By holding a display in your hand, one can interact with it in several gestural ways to change the displayed view and visualization parameters. The support of touch and pen interaction directly on the handheld display allows for additional interactivity.

2. Enhance common $2 D$ interaction with additional $3 D$ interaction. The usage of a graspable display that can be moved freely in 3D space implies a very natural way of interaction based on the metaphor of looking at pictures or documents.

3. Replace virtual views by physical, tangible views. Tangible views provide additional physical display space that can be utilized to support multiple coordinated views, overview \& detail as well as for focus + context techniques.

The main contribution of this paper is a conceptual investigation of tangible views in the context of information visualization. We start by an analysis of related work, followed by a description of properties and degrees of freedom of tangible views as a tool of both representation and interaction. Subsequently, the applicability of tangible views to a variety of information visualization solutions is illustrated with several cases studies. Thereby, we demonstrate that tangible views are an interesting alternative to classic interaction and that they can be used for novel kinds of interaction that are more natural and intuitive compared to traditional input and output devices. We continue with a discussion of early user feedback and possible limitations. Later, technical aspects of the system are briefly described. Finally, we close with a reflection of our approach and indicate directions for future work and potential applications of tangible views.

\section{RELATED WORK}

\section{Conventional Interactive Visualization}

Conventional information visualization solutions address desktop computers with a single virtual desktop (possibly one that spans multiple stationary displays) and standard input devices (e.g., mouse, track-ball, keyboard). One or multiple virtual views are shown that provide different visualizations of the data under investigation. Common use cases for multiple views are to provide overview and detail or to compare alternative visual encodings or different parts of the data [3].

To accomplish exploration tasks, the interactive adaptation of the visualization to the task and data at hand is crucial. Yi et al. identified several high-level user intents for interaction [46]. Users want to mark something as interesting, e.g. specific data items by brushing [4]. For exploratory analyses, users also need to alter views. This can be achieved by navigating the view space $[6,41]$ or the data space [35], or by using common user interface controls to adjust the visual encoding and to rearrange views on the virtual desktop. Particularly for larger data sets it is necessary to filter the data interactively [2] and to switch between different levels of abstraction [10]. For higher order visualization tasks users often need support for relating and comparing data items $[11,36]$.

Technically, any interaction can be modeled as adjustments of visualization parameters [20]. With direct manipulation [30], users interact directly with the visual representation. Physical movement of pointing devices is translated into specific interaction semantics, for instance, to select data items of interest (see $[13,15])$ or to transform the view on the data (see $[14,9])$. Indirect manipulation uses control elements, such as sliders, to adjust numeric visualization parameters or to filter out data items that are irrelevant.

A special class of techniques are virtual lenses [5]. Lenses combine different visualization and interaction concepts in one interactive tool. Lenses exist that magnify interesting items or regions [28], that filter represented information [8], that rearrange visualized data items [36], or that adjust the visual encoding [34]. The diversity of lens techniques indicates that they are a universal tool to support most of the user intents identified by Yi et al. [46]. Generally, a lens is defined as a spatially confined sub-region of the display and a lens-specific visual mapping. By means of direct manipulation, users can move the lens to specify the part of the visual representation that is to be affected by the lens mapping. 


\section{Towards More Direct Interaction}

Direct manipulation in information visualization can be accomplished with indirect pointing devices, such as the prevalent mouse, where the input space does not coincide with the display space. Direct input, by contrast, unites the interaction and display space and is often performed using a digital pen or finger on touchscreens. An enhancement is multi-touch technology that allows users to execute commands by performing complex gestures with multiple fingers on the display surface simultaneously. Even though natural direct manipulation concepts lends themselves to the field of information visualization, the mouse still dominates the field.

Approaches that investigate direct or tangible interaction in information visualization are scarce. Isenberg and Carpendale explicitly make use of interactive tabletop displays for the purpose of performing comparison tasks [17]. Via direct interaction on the tabletop, users can compare aspects of tree representations. Isenberg and Fisher apply multi-touch technology to support collaborative visualization tasks [18]. The iPodLoupe introduced by Voida et al. [42] goes one step further and adds a physical local display to the visualization environment. While a large interactive tabletop display shows the visualization context, a small focus display (iPod) is used to show details. Yet, the interaction remains on the tabletop display; users cannot interact by manipulating the focus display in space.

The traditional visualization methods reviewed above are mostly using indirect input and are based on virtual views, i.e., windows on a physical display or local views embedded into the visualization. Spatially aware displays, which know precisely about their position and orientation in 3D space, are a promising approach to make virtual views physically touchable and to accomplish direct and natural interaction.

A pioneer work in making virtual views physically tangible is the metaDESK system by Ullmer and Ishii [38]. The system consists of a tabletop display and an LCD panel that is attached to the tabletop via a mechanical arm. By moving around the LCD panel users can navigate through polygonal 3D models. Yee's Peephole Displays [45] support the interaction with a digital desktop environment (calendar, web browser, street map) that is virtually wrapped around the user. A prominent example of a paper-based passive display are Paper Windows by Holman et al. [16], which support various ways of interacting with a graphical user interface. Sanneblad and Holmquist used spatially aware monitors to magnify details of a street map that is displayed on a large stationary vertical display [27]. In [26], Molyneaux et al. present a technical architecture for bi-directional interaction with tangible objects (input/output), similar as proposed in our work. However, their discussion is mostly on technical aspects and focuses only briefly on modalities of interaction. To allow for simultaneous back-projection of different contents onto a tabletop surface and a tangible lens, respectively, Kakehi and Naemura use a special projection foil that changes its translucency depending on the projection angle [21]. The SecondLight system by Izadi et al. [19] supports dual projections by using electronically switchable diffusers. The PaperLens [32] is a technically less complex combination of a tabletop context display and a spatially aware lightweight focus display. The system allows users to explore spatial information spaces simply by moving the lightweight display through the physical 3D space above a tabletop surface.

\section{Closing the Gap}

In summary, we see a twofold gap. On the one hand, information visualization strives for natural direct manipulation with the visual representation and the data, but only few approaches utilize the available technologies to this end. On the other hand, various approaches have been developed to support direct interaction with lightweight physical displays, but none of them addresses the specific representational and interaction aspects of information visualization.

Our aim is to narrow this gap by means of tangible views. The work we present here builds upon the previous PaperLens system, where a tabletop display provides the contextual background for the exploration of spatial information spaces with a spatially aware tangible magic lens [32]. Four different classes of information spaces were identified and are supported by the system: layered, zoomable, temporal, and volumetric information spaces. While horizontal translation ( $x-y$ position on or above the tabletop) is reserved for panning operations, lifting or lowering the magic lens enables users to choose from a set of two-dimensional information layers, to perform zooming of a high-resolution image, to go forward or backward in time in a video, and to explore the third dimension of a volumetric data set. Thanks to this explicit mapping of the magic lens' $z$-position (height above the tabletop) to the defining characteristics of each data class, users experienced the exploration of these information spaces as intuitive and natural. This motivated us to use PaperLens as the basis for our work.

In this paper, we technically and conceptually extend this approach in the following key points: (1) generalization of the interaction vocabulary including novel gestures and support for multiple tangible views, and (2) mapping of the vocabulary to semantics specific to information visualization.

\section{TANGIBLE VIEWS}

In this section, we will systematically investigate tangible views as a class of devices that serves two purposes at the same time: as a tool of representation and as a tool of interaction. We begin our discussion by focusing on the general characteristics and illustrate what is syntactically possible when using tangible views. In the next section, we will add semantics to these possibilities by mapping them to tasks that are common in the field of information visualization.

\section{Tool of Representation}

In its simplest form, a tangible view is a spatially aware lightweight display or projection surface onto which arbitrary information can be projected. Tangible views usually do not exist on their own, but instead are integrated into an environment of one or more stationary displays of arbitrary size, shape and orientation. By displaying graphical information, these stationary displays or surfaces both define and provide the contextual background of a virtual information world in which a tangible view exists. A basic display configuration will be used throughout this paper: a horizontal 


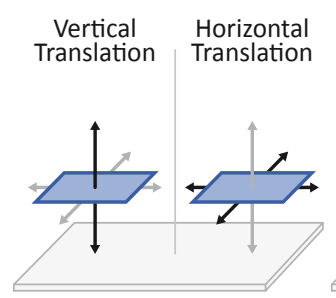

(a) Translation

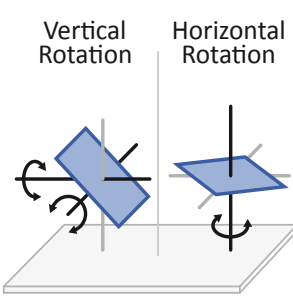

(b) Rotation

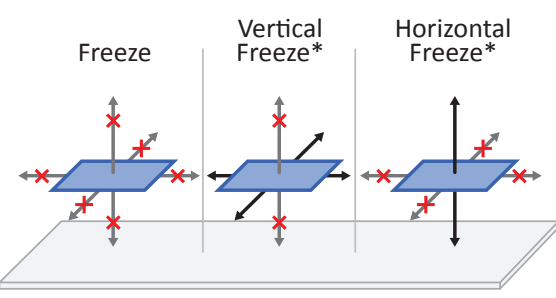

(c) Freezing

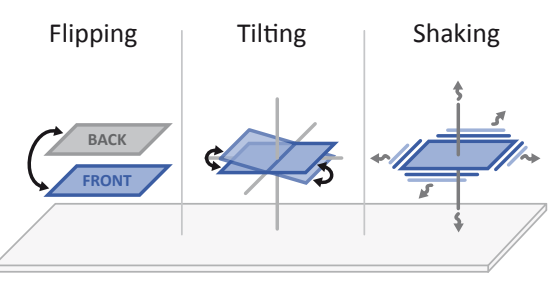

(d) Gestures

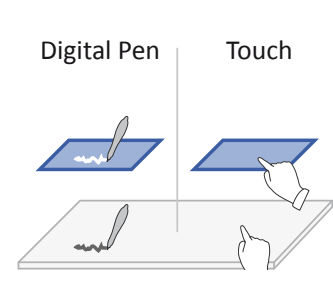

(e) Direct Pointing

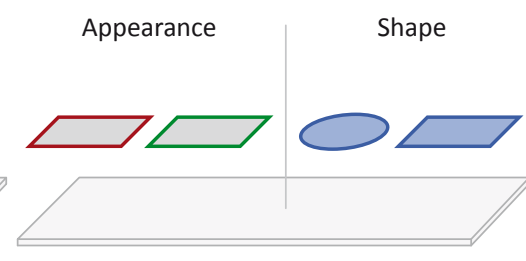

(f) Toolbox Metaphor

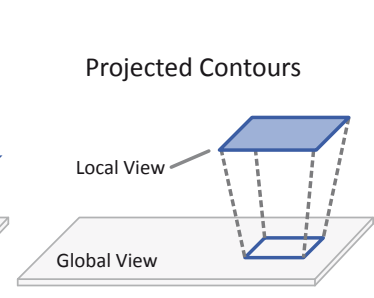

(g) Visual Feedback

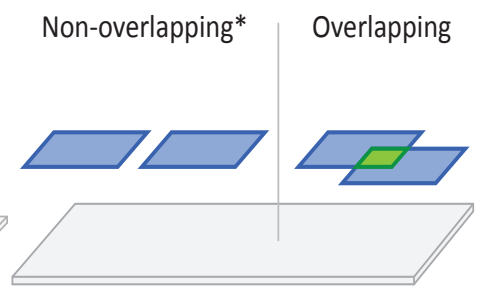

(h) Multiple Views

Figure 2: Overview of the interaction vocabulary of tangible views (asterisks denote novel techniques).

tabletop whose purpose is to serve as the main context view and tangible views as local views into the information space. This thinking relates to the focus + context concept.

One important advantage of tangible views is that they can be used with other tangible views simultaneously. Thus, they can be understood as a multiple view environment with each tangible view representing a unique physical view into a virtual information world. This characteristic makes them an ideal tool for collaboration or comparison tasks and for supporting the overview and detail approach. Besides that, tangible views usually appear in different shapes and sizes. Most commonly a tangible view will be of rectangular or circular shape, but other more sophisticated shapes, like hexagonal or metaphorical shapes (e.g., "magnifying glass"), are possible and may play a special role during interaction.

\section{Tool of Interaction}

Throughout our investigations of the various aspects of tangible views, we aimed at as-easy-to-learn and as-natural-aspossible usage that is inspired by everyday life interaction principles. Interacting with tangible views is basically as simple as grabbing a lightweight physical object (the tangible view) with one or both hands and then moving it around in the real-world space, while the tangible view constantly provides appropriate visual feedback. The actual interaction takes places within the physical space that is defined by the stationary display that serves as the contextual background. In our case, the space above the horizontal tabletop's surface is used as the three dimensional reference system that we refer to as the interaction space. Despite previous research on interacting with non-rigid tangible screens, such as foldable [25] or bendable [29] approaches, we restricted our investigations on rigid tangible displays only.

As with all rigid objects in a 3D space, there are six degrees of freedom (6DOF) available for interaction. More precisely, the basic degrees of freedom are the position $(x, y$, and $z)$ with respect to the interaction space and the local orientation of the tangible view $(\alpha, \beta$, and $\gamma)$. Corresponding interactions are translation and rotation, respectively. Both are very easy to learn and simple to execute. Additionally, interaction can be enhanced by introducing higher level interaction gestures (on the basis of basic degrees of freedom). Such gestures enrich the interaction vocabulary of users and thus can make it easier for them to solve particular sets of problems.

It is important to note that the ways of interaction that we discuss here are similar to those in the field of tangible interaction, where "graspable" objects represent specialized tools that can be used to physically interact with a display surface, in particular tabletops. However, there are three major differences between tangible and tangible view interaction. First, traditional tangible interaction is limited to the tabletop surface itself, whereas the usage of the space above it is rarely seen with the Multi Layer Interaction for Digital Tables by Subramanian et al. [33] being a minor exception. By contrast, with tangible views we propose a technique that utilizes the space above a tabletop explicitly for the purpose of interaction. Second, tangibles usually are characterized by specialized form factors or well-defined shapes that make them fit perfect for a particular task or set of tasks, e.g. for adjusting parameters such as in SLAP Widgets by Weiss et al. [43]. On the contrary and although tangible views can come in various shapes too, they provide a much more generic and multipurpose way of interaction. This is probably due to the third important difference: tangible views provide a constant visual feedback and thus their appearance is customizable. This is a feature that traditional tangibles lack or at least provide very seldom or only in a limited way.

\section{Interaction Vocabulary}

The design space for tangible views is more complex and richer than it looks at a first glance. Therefore, some fundamental principles need to be found and understood that help both users and system designers. In this respect, many interaction techniques, such as gestures, have been described and used previously. Our intention was to organize, combine, and extend these ideas in a meaningful way and with focus on tai- 
loring them towards the domain of information visualization. This was one goal of our research and as a result we identified the following eight basic usage patterns for tangible views: translation, rotation, freezing, gestures, direct pointing, the toolbox metaphor as well as multiple views, and visual feedback. The first six patterns are mainly motivated by the available degrees of freedom and additional interaction modalities, and thus are features of the "tool of interaction". In contrast, the last two patterns (visual feedback, multiple views) are motivated by properties of the "tool of representation". In the following, we will discuss these eight patterns for tangible views in more detail.

Translation. One way of interacting with a tangible view is to interpret its current 3D position and thus to utilize shifts of movement as a form of interaction [32]. The resulting three degrees of freedom (3DOF) can then be interpreted either by utilizing all 3DOF at the same time or by restricting them to one or two axes: horizontal translation as movement in the $x$ - $y$-plane and vertical translation as movement along the $z$-axis (see Figure 2(a)).

Rotation. Another way of interacting with a tangible view is to use its local orientation, i.e., changes of $\alpha, \beta$, and $\gamma$ (3DOF). Without the claim of completeness, we distinguish between two types of rotation: horizontal rotation [23] around $z$ and vertical rotation [25] as rotations around $x$ and/or $y$. This is illustrated in Figure 2(b).

Freezing. In certain situations, it is necessary to move a tangible view without the intention of interacting with the system. This happens, for example, when users want to study a specific view in more detail or when they want to keep it for later examination by physically placing the view on or beside the table surface. For this purpose, we introduce the possibility of freezing a tangible view (see Figure 2(c)). In terms of degrees of freedoms used for interaction, this means that the system ignores shifts of movement for all or some principle axes. We distinguish between three different freezing modes: normal freeze [31] where $x-y-z$ are locked, vertical freeze where only $z$ is locked, and horizontal freeze where only $x-y$ are locked. Hereby, the latter two techniques are new to the field.

Gestures. So far, we used the available 6DOF in a very direct manner. But there is room for more complex types of interactions by using the concept of gestures. In order to enrich the interaction with tangible views, we propose the use of following (non-exhaustive) set of simple gestures: flipping [16], shaking [44], and tilting [7] (see Figure 2(d)). The principle idea of flipping is to attach different meanings to the front and the back side of a tangible view and thus to let users interact with the system by turning a tangible view around. As the name implies, shaking lets users interact with the system by arbitrarily moving a tangible view to and fro. In contrast, sideways and frontways tilting is like slanting the tangible view slightly to the left/right (around the $y$-axis) and to the front/back (around the $x$-axis), respectively.

Direct Pointing. Direct pointing borrows its ideas from the fact that in addition to interacting with tangible views, it is also possible to perform interaction on them by provid- ing further methods of input. Without loss of generality, we distinguish between multi-touch and digital pen for interacting on both the tangible views and the context display (see Figure 2(e)). These technologies allow users to interact with a visual representation by means of direct pointing. Thumb movements or touch, for instance, can be recognized to control context-sensitive user interface elements on tangible views. Digital pens are utilized for more precise input such as for writing or exact pointing [31].

Toolbox Metaphor. The main idea of the toolbox metaphor is to assign specialized tasks to the physical properties [12, 39] of tangible views. In particular the shape (e.g., circle or rectangle) and the visual appearance (e.g., color or material) of a tangible view are relevant. As hinted in Figure 2(f), these properties can be used as a basis to decode certain tasks or tools by the physical look of a tangible view. Following this concept, a set of pre-manufactured tools (tangible views) is presented in close proximity to the tabletop. Depending on their aim of interaction, users can then easily select the appropriate tool for a particular problem by simply grabbing another tangible view.

Visual Feedback. Visual feedback is a fundamental requirement for the interaction with a visual system such as tangible views. When interacting with tangible views, users expect instant visual feedback in correspondence with the interaction. Such feedback is provided directly on a tangible view or on the stationary tabletop display. Visual feedback also serves to illustrate the interplay of views by projecting the tangible view's contour lines onto the tabletop surface [32] (see Figure 2(g)).

Multiple Views. As depicted in Figure 2(h), tangible views support the concept of multiple local views within the reference system of a global view. We distinguish between nonoverlapping and overlapping local views. We define the term overlapping as whether two or more tangible views consume the same or partly the same horizontal $(x-y)$ space above the tabletop (by ignoring the $z$-axis). In our understanding, overlapping tangible views can influence each other, i.e., the visual output of one tangible view may depend on the output of another one. In contrast, non-overlapping tangible views are independent from each other. In combination with freezing, multiple views provide the foundation for several two-handed comparison techniques as described in the next section. To the best of our knowledge, such tangible comparison techniques have never been presented before.

\section{CASE STUDIES}

From the previous section we see that tangible views provide a rich vocabulary that comprises interaction aspects (tangible) and representation aspects (view). This section will address the question of how this vocabulary can be applied to information visualization. Our discussion begins with some general considerations. Then, we explain how tangible views can support users in accomplishing common interaction tasks. For this purpose, we have implemented five visualization approaches that demonstrate the versatility of tangible views. 


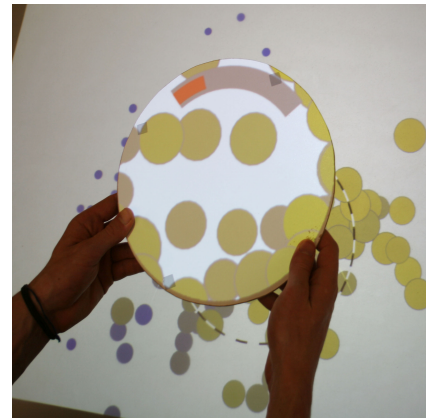

(a) Low degree of displacement.

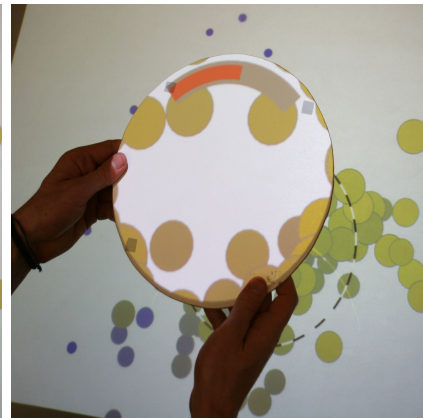

(b) Higher degree of displacement.

Figure 3: Scatter plot: A circular fisheye lens allows users to control the parameters lens location and degree of magnification by using horizontal and vertical translation, respectively. The fisheye-lens' degree of displacement is adjusted by horizontal rotation.

\section{General Considerations}

Traditional visualization techniques address a two-dimensional presentation space that is defined by the axes of the display. In contrast, with tangible views we extend the presentation space by a third axis - the z-axis that emanates perpendicularly from the tabletop surface. The motivation for the extension to the third dimension lies in the data-cube-model with the space above the tabletop display being the physical equivalent of an otherwise virtual data-cube. This allows us to project data not only onto the tabletop's surface, but also into the space above it. As we will see in the following case studies, there are various options how to utilize the additional dimension for interaction and visualization purposes.

Here, two fundamental aspects are multiple view visualizations (that provide different visual representations simultaneously [3]) and lens techniques (local views with a specific visual encoding embedded into a visualization context). As any tangible view functions as a physical window into virtuality, multiple views and lenses can easily be made tangible. Beyond that, direct manipulation is naturally supported by tangible views as well: users can move a tangible view around to specify the area or the data items to be affected by the lens.

\section{Case Study: Graph Visualization}

Node-link-diagrams and hierarchical abstraction are classic means to enable users to interactively explore large graphs. Starting at the abstraction's root, users expand or collapse nodes in a series of discrete interactions until information density suits the task at hand. A continuous navigation through the different levels of abstraction has been introduced by van Ham \& van Wijk [40]. We implemented a tangible variant of such an abstraction lens and applied it to explore relations in the ACM classification. As demonstrated in Figure 1(a), a rectangular tangible view serves as a local abstraction view for the graph that is being shown on the tabletop display. Users can naturally pan the view by using horizontal translation and freely change the degree of detail by vertical translation. This way it is possible to quickly explore different parts of the graph and compare relations at different scales. At all times, the tabletop display provides
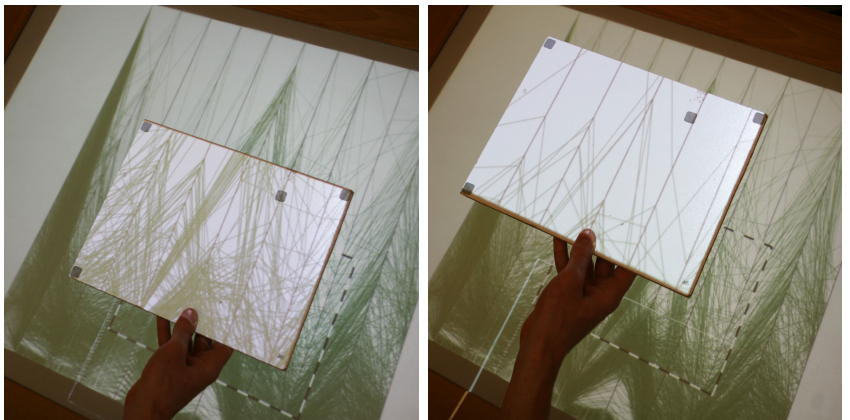

(a) Every 32nd polyline of the origi- (b) Every 256th polyline of the orignal dataset is displayed.

inal dataset is displayed.

Figure 4: A tangible sampling lens supports users in finding an appropriate sampling factor by using vertical translation. Projected outlines on the tabletop helps users mentally linking local and global views. visual feedback about the current positions of the local view within the global view.

\section{Case Study: Scatter Plot}

Scatter plots visualize correlation in a multivariate data set by mapping two variables to $\mathrm{x}-\mathrm{y}$ position of graphical primitives, where color, size, and shape of these primitives can be used to encode further variables. However, graphical primitives could become very tiny and could overlap or occlude each other, which impedes recognition of color and shape. To make size, color, and shape of the data items discernible, our scatter plot implementation is equipped with a graphical zoom lens and a simple fisheye lens, which temporarily sacrifices the positional encoding to disentangle dense parts of a scatter plot. According to the toolbox metaphor, a rectangular tangible view and a circular tangible view represent the zoom lens and the fisheye lens, respectively. The tabletop display serves as the visual context showing two data variables (out of four of the well-known IRIS data set) mapped onto the tabletop's $x$ and $y$-axis, respectively. Users can easily alternate the variables to be visualized by using frontways $(x$-axis) and sideways tilting ( $y$-axis). Horizontal translation is again applied to set the lens location and vertical translation controls the geometric zooming factor for the zoom lens. The degree of displacement for the fisheye lens is manipulated with horizontal rotation. During this latter interaction, a curved slider on the view's surface provides visual feedback of the current parameter value (see Figure 3).

\section{Case Study: Parallel Coordinates Plot}

Classic parallel coordinates encode multiple quantitative variables as parallel axes and data items as polylines between these axes. This encoding is useful when users need to identify correlated variables or clusters in the data. However, as the number of data items increases, parallel coordinates suffer from cluttering. Ellis and Dix suggest using a sampling lens to mitigate the problem [8]. As sampling is often random, it is not clear in advance, what a good sampling factor is. We implemented a tangible sampling lens (see Figure 4) that supports users in interactively finding a suitable sampling factor. While the background visualization shows the whole dataset (11 variables and 1100 records of a health- 

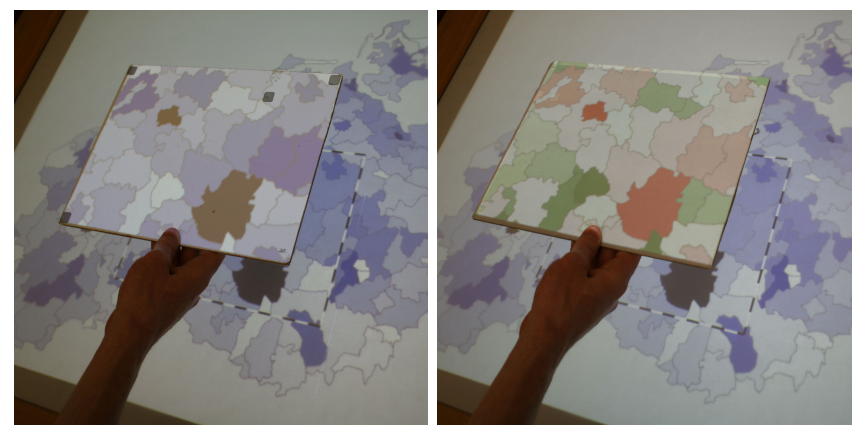

(a) Before flipping: visualization (b) After flipping: visualization supsupporting the task of identification. porting the task of localization.

Figure 5: By flipping a tangible view, users can choose between visualizations that support different tasks.

related dataset), the tangible lens shows only every $i$-th data item. Analogous to the previous case studies, the lens location is set by horizontal translation. By vertical translation, users can traverse through possible values for $i$ (degree of sampling). For the purpose of demonstration, our basic prototype simply uses $i \in(1,2,4,8,16 \ldots)$. Beyond that, attribute axes of the parallel coordinates plot can be reordered with direct pointing by using digital pens (Anoto). Axes rearrangements can be performed on both tangible view and tabletop.

\section{Case Study: Matrix Visualization}

Yi et al. [46] list visual comparison as an important interaction intent that involves various steps, as for instance, selecting subjects for the comparison, filtering the data to compare specific subjects only, or encoding of additional information to support the comparison. Performing visual comparison with traditional means is usually difficult due to the numerous heterogeneous interactions participating. On the other hand, direct interaction on a tabletop can facilitate comparison [17]. How tangible views can be applied for visual comparison will be illustrated next. For the sake of simplicity, we use rectangular tangible views and a matrix visualization of a synthetic graph (42 nodes and 172 edges) that is displayed on the tabletop display as shown in Figure 1(b). In the first phase of comparison, tangible views are used to select data subsets. By horizontal and vertical translation users can determine position and size of a subregion of the matrix and then freeze the selection. Once frozen, the user can put the tangible view aside and take another one to select a second data subset. The two frozen tangible views can now physically be brought together either by holding each one in a separate hand or by rearranging them on the tabletop. As additional visual cues, smooth green and red halos around compared data regions indicate similarity and dissimilarity, respectively. If a selection is no longer needed it can be deleted by the shaking gesture.

\section{Case Study: Space-Time-Cube Visualization}

Space-time-cubes are an approach to integrate spatial and temporal aspects of spatio-temporal data in a single visual representation [22]. The analogy between a space-time-cube and the three-dimensional presentation space used for tangible views motivated this case study: the tabletop display's $x$ and $y$-axis show the spatial context as a geographic map,

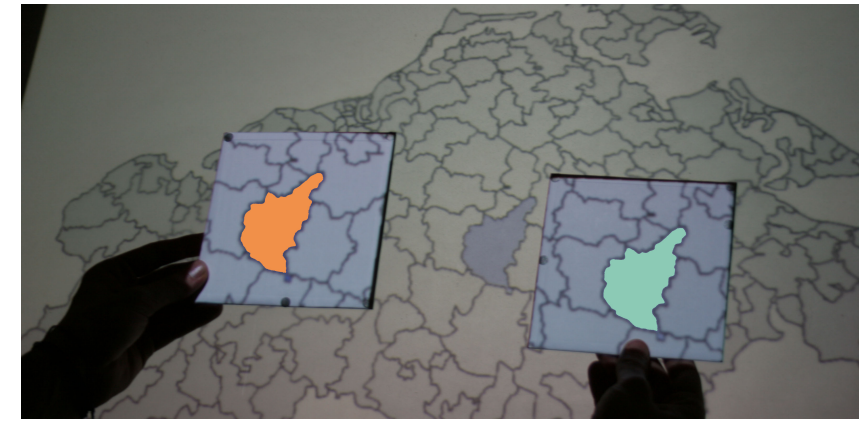

Figure 6: After locking the focus of two tangible views to the same location by horizontally freezing, users can visually compare between the two views by lifting or lowering them simultaneously.

and the dimension of time (12 months) is mapped along the $z$-axis.

Tangible views are used as physical viewports into the spacetime-cube. Interactive exploration is driven by horizontal and vertical translation to navigate the map and the time axis, respectively. When held in a horizontal orientation, a tangible view shows the data for a selected month, i.e., a horizontal slice through the space-time-cube. To get an overview for all months (i.e., a vertical slice), users can rotate a tangible view into upright orientation. Then the visual representation changes to a simple color-coded table that visualizes multiple variables for all 12 months for the area above which the tangible view is hovering (see Figure 1(c)). Depending on whether the user's task is to identify data values or to locate data values, different color schemes are used to encode data values (see Figure 5) [37]. Simply flipping the tangible view switches between both tasks.

Exploring spatio-temporal data usually involves comparing different areas, different time steps, or both in combination. Freezing a tangible view helps users to accomplish these goals more easily. With vertical freeze, a tangible view can be locked to a certain month, effectively unlinking vertically translation and navigation in time. When frozen, the tangible view can even be put down to relocate the entire interaction to the tabletop surface itself. This can be quite useful for handling multiple views simultaneously in order to compare attributes between different areas, or for marking a certain detail for later examination by simply leaving the tangible view on a particular area. Horizontal freeze lets users lock a tangible view to a certain area. This is useful for comparing different months of the same location. To this end, the user simply locks two tangible views onto the same area. It is then possible to lift or lower the two views independently to compare two months, while the horizontal freeze guarantees that the focused area does not change unintentionally (twohanded comparison, see Figure 6).

\section{DISCUSSION}

From designing the case studies and initial user feedback, we crystallized a set of observations that may be useful guidelines for further more complex applications. We also discuss potential limitations and critical comments of users. 


\section{Observations}

Based on the case studies, we derived following observations: I. Providing direct visual feedback, such as cast shadows of tangible views on the tabletop, helps users mentally linking local and global views. II. Translation should be reserved for navigation in presentation space. III. Freezing is essential to temporarily decouple a tangible view from one or multiple axes of the interaction space. This is necessary to support tasks that require rearrangement of views, most prominently, comparison tasks, but also helps switching to traditional interaction, such as multi-touch. IV. Direct pointing is essential for interacting within local or global views (tangible or tabletop). It is a requirement for precise selection tasks. V. By favoring orthogonal interaction (e.g., shape for choosing a tool, translation for navigating the presentation space, horizontal rotation for navigating the parameter space, and tilting for navigating the data space), users can implicitly communicate their intent to the system without the need of explicitly changing any global interaction states.

\section{Limitations}

We have shown the case studies to a variety of users and generally received positive feedback. Even domain experts, at first reluctant, were quickly convinced of the techniques after seeing a live demo. Interestingly, before testing the demo and by only knowing the theoretical concept, some of them suspected that it would be "too tiring to hold and move the tangible views through the air" if compared to "use a stylus on a tabletop, where users can rest their elbow on the surface". Although this is true for extensive use, users commented that the mix of tangible interaction and the use of more traditional pen or touch input, e.g., after freezing a tangible view and laying it down on the table, reduced this problem considerably. In general, users did not have problems with lifting up the tangible views too high, because we restricted the physical interaction volume to $40 \mathrm{~cm}$ above the table. Thus, users were able to find boundaries of the interaction volume quite easily (no visual feedback above a certain height). In some cases, we also provided additional navigational aids, such as height indicators inspired by [32]. Users felt that this was helpful for finding certain layers of information more efficiently. In addition, the system allows to tilt lenses slightly in order to prevented viewing angle becoming too glancing. Sometimes, users complained about problems with precise interaction and hand tremor when moving or rotating tangible views in order to adjust an accurate position or angle. Here, convincing solutions need to be found and evaluated, which is beyond the scope of this paper. Also, one user suggested to provide better support for letting users know which actions are mapped to what. Similar to traditional GUI widgets, labels and tooltips could reveal what the widget does or even show that there is an affordance. The same user remarked that "each tangible view has a fixed size and shape, unlike standard windows in a GUI". This could be tackled by having a collection of different sized tangible views or even by future hardware that allows unfolding of displays, similar to [25]. Despite of these issues, we are very confident that tangible views are a very powerful and generic technique that paves the way for an exciting field of research with many challenging questions.

\section{TECHNICAL SETUP}

For the technical setup of tangible views we extended the PaperLens approach by Spindler et al. [32], particularly in terms of tracking technology, gesture recognition, and direct input techniques (digital pens and touch) for both tangible views and the tabletop. The setup consists of a tabletop display as well as several infrared (IR) cameras and a top projector that are attached to the ceiling. This setup is enriched with various tangible cardboard displays (tangible views) that can be freely moved through the space on or above the tabletop.

In order to bring such a system alive, several problems need to be solved: tracking of tangible views to make them spatiallyaware, displaying image content, recognizing gestures, support for direct pointing, as well as providing application functionality. Many of these tasks can be tackled independently from each other and thus have been split up (on a technical basis) between different computers. Hereby, for the purpose of inter-computer communication, we use public protocols for streaming device states (VRPN) and remote procedure calls (XML-RPC).

Tracking. The problem of determining position and orientation of tangible views is solved by tracking. Various tracking approaches have been used in the past, such as mechanical (arm-mounted) [38], ultrasonic [27], and optical solutions with visible markers [24]. However, a major design goal of PaperLens [32] is to conceal the technical aspects from users as much as possible (no cables, no disturbing markers, etc.). This has been accomplished by sticking small, unobtrusive IR-reflective markers ( $4 \mathrm{~mm})$ to the corners/borders of tangible views. These markers can then be tracked by Optitrack FLEX:V100R2 cameras. As opposed to the original PaperLens implementation that only uses one camera and a simple home-made tracking, we extended the system by using six cameras and a commercially available tracking solution (Tracking Tools 2.0) that is more accurate and allows for defining arbitrary marker combinations. These are used to encode lens IDs (for the toolbox) as well as front and back sides of lenses.

Displaying Image Content. Tangible views are implemented as a passive display solution, i.e., image content is projected from a ceiling-mounted projector onto an inexpensive lightweight projection medium (cardboard or acrylic glass). This allows for a low-cost production of tangible views in various sizes and shapes (rectangles, circles, etc.) and also includes control of the visual appearance (color, material) as well as using the tangible views' back sides as displays. Once position and orientation of a tangible view is known, this information is fed to a computer. Thus, the connected top projector can project arbitrary image content onto the tangible view. In order to maintain a perspectively correct depiction of the image content, OpenGL is used to emulate the physical space above the tabletop including all tangible views that reside there. The OpenGL camera is precisely located at the virtual position of the top projector and the physical properties of lenses are represented by textured polygons (shape, position, and orientation). Image content is then rendered independently into FrameBufferObjects (FBO) that are attached to these textures. In this way, application code is sep- 
arated from the more generic projection code. This will allow us to easily exchange the top-projected passive displays with lightweight active display handhelds in the near future.

Recognizing Gestures. In order to support flipping, shaking, and tilting, a simple gesture recognizer has been implemented. Flipping is recognized with the help of unique markers that identify front and back side of a tangible view. For other gestures, we identified characteristic movement patterns that can be detected by the system, i.e., for shaking a rapid irregular movement with small extent and for tilting a back-and-forth rotation along an axis in a range of about $20^{\circ}$.

Direct Pointing. In terms of interacting on tangible views, the system was augmented with support for direct pointing, in particular touch and digital pens. Digital pen technology can easily be incorporated by gluing Anoto-paper [1] onto a tangible view's surface. The Anoto paper shows a unique dot pattern that is scanned by special pens with a built-in camera for determining their position on the lens's surface. This $2 \mathrm{D}$ position is then transmitted to the application via Bluetooth in real-time. The system was further enhanced with basic support for touch input. For this purpose, additional IRreflective markers have been affixed to the surface of tangible views. By hiding these "marker buttons" with their thumbs, users can activate certain states, such as the freeze mode.

\section{CONCLUSION}

Conventional desktop display solutions and indirect interaction by means of traditional input devices are notable limitations for information visualization. To overcome these limitations, we introduced tangible views, which integrate display and interaction device. Tangible views provide additional display space and allow for a more natural and direct interaction. They serve as a viewport into a 3D presentation space and utilize the additional axis for various interaction and visualization purposes. To the best of our knowledge, this is the first time that spatially-aware displays were employed in the field of information visualization.

In this paper, we composed a versatile set of orthogonal interaction patterns serving as a basic vocabulary for carrying out a number of important visualization tasks. Users can perform a variety of gestures directly with a tangible view, or use touch and pen input on a tangible view. Tangible views provide haptic affordances combined with clear proprioception by means of body movements. At the same time, we are employing the well-known metaphors of moving sheets of paper on a desk as well as lifting photos and other documents to look at them in detail. As previous studies suggest [32], interaction with tangible views is perceived as very natural.

We see the true potential of our approach in the possibilitiy to provide interesting alternatives to classic techniques and to supersede virtual views by physically tangible ones. With that, fairly direct mappings can be achieved for multiple coordinated views, overview \& detail techniques, and focus + context techniques, in particular lens techniques. In addition, bimanual interaction allows for the natural control of various visualization parameters in parallel, which cannot be accomplished with traditional desktop interfaces. Here, we contributed two-handed comparison techniques. By means of the toolbox metaphor, we can utilize tangible views to facilitate task-oriented visualization, which resembles the usage of physical workshop or kitchen tools.

Future Work. The very positive early user feedback we received suggests that the application of tangible views for information visualization tasks is a very promising approach. However, further thorough studies of particular combinations of tangible views and visualization techniques are required. For that, we need to refine the interaction techniques, especially with regard to touch input and parameter controls. In addition to the already investigated and mentioned applications of tangible views, there are further visualization challenges that may benefit from our approach, among them interactive visual assistance for data fusion with tangible view and collaborative problem solving supported by tangible views. With tangible views, we hope to have made a contribution especially to the interaction side of information visualization and to stimulate a discussion on more natural ways of looking at and interacting with data.

ACKNOWLEDGMENTS. We thank Michel Hauschild for the great help in implementing the system and Ricardo Langer for his artwork and video editing. Our work was funded by the "Stifterverband für die Deutsche Wissenschaft" and by the German Ministry of Education and Science (BMBF) within the ViERforES project (no. 01 IM 08003).

\section{REFERENCES}

1. Anoto Group AB. http://www.anoto.com.

2. C. Ahlberg, C. Williamson, and B. Shneiderman. Dynamic Queries for Information exploration: An Implementation and Evaluation. In Proc. of CHI, pp. 619626, 1992.

3. M. Q. Wang Baldonado, A. Woodruff, and A. Kuchinsky. Guidelines for Using Multiple Views in Information Visualization. In Proc. of AVI, pp. 110-119, 2000.

4. R. A. Becker and W. S. Cleveland. Brushing Scatterplots. Technometrics, 29(2):127-142, 1987.

5. E. A. Bier, M. C. Stone, K. Pier, W. Buxton, and T. D. DeRose. Toolglass and Magic Lenses: The SeeThrough Interface. In Proc. of SIGGRAPH, pp. 73-80, 1993.

6. A. Cockburn and J. Savage. Comparing SpeedDependent Automatic Zooming with Traditional Scroll, Pan and Zoom Methods. In British HCI 2003, pp. 87102, 2003.

7. R. Dachselt and R. Buchholz. Natural Throw and Tilt Interaction between Mobile Phones and Distant Displays. In Extended Abstracts of CHI '09, pp. 32533258. ACM, 2009.

8. G. Ellis and A. Dix. Enabling Automatic Clutter Reduction in Parallel Coordinate Plots. IEEE TVCG, 12(5):717-724, 2006.

9. N. Elmqvist, P. Dragicevic, and J.-D. Fekete. Rolling the Dice: Multidimensional Visual Exploration using Scatterplot Matrix Navigation. IEEE TVCG, 14(6):1141-1148, 2008.

10. N. Elmqvist and J.-D. Fekete. Hierarchical Aggregation for Information Visualization: Overview, Techniques, and Design Guidelines. IEEE TVCG, 16(3):439-454, 2010. 
11. N. Elmqvist, Y. Riche, N. Henry-Riche, and J.-D. Fekete. Melange: Space Folding for Visual Exploration. IEEE TVCG, 16(3):468-483, 2010.

12. G. W. Fitzmaurice. Graspable User Interfaces. $\mathrm{PhD}$ thesis, University of Toronto, Canada, 1996.

13. H. Hauser, F. Ledermann, and H. Doleisch. Angular Brushing of Extended Parallel Coordinates. In Proc. of IEEE InfoVis, pp. 127-130, 2002.

14. K. Henriksen, J. Sporring, and K. Hornbæk. Virtual Trackballs Revisited. IEEE TVCG, 10(2):206-216, 2004.

15. H. Hochheiser and B. Shneiderman. Dynamic Query Tools for Time Series Data Sets: Timebox Widgets for Interactive Exploration. Information Visualization, 3(1):1-18, 2004.

16. D. Holman, R. Vertegaal, M. Altosaar, N. Troje, and D. Johns. Paper Windows: Interaction Techniques for Digital Paper. In Proc. of CHI, pp. 591-599, 2005.

17. P. Isenberg and M. S. T. Carpendale. Interactive Tree Comparison for Co-located Collaborative Information Visualization. IEEE TVCG, 13(6):1232-1239, 2007.

18. P. Isenberg and D. Fisher. Collaborative Brushing and Linking for Co-located Visual Analytics of Document Collections. Computer Graphics Forum, 28(3):10311038, 2009.

19. S. Izadi, S. Hodges, S. Taylor, D. Rosenfeld, N. Villar, A. Butler, and J. Westhues. Going Beyond the Display: A Surface Technology with an Electronically Switchable Diffuser. In Proc. of UIST, pp. 269-278, 2008.

20. T. J. Jankun-Kelly, K.-L. Ma, and M. Gertz. A Model for the Visualization Exploration Process. In Proc. of IEEE VIS, pp. 323-330, 2002.

21. Y. Kakehi and T. Naemura. UlteriorScape: Interactive Optical Superimposition on a View-Dependent Tabletop Display. In Proc. of Workshop on Horizontal Interactive Human Computer Systems, pp. 189-192, 2008.

22. M. J. Kraak. The Space-Time Cube Revisited from a Geovisualization Perspective. In Proc. of the 21st Intern. Cartographic Conference, pp. 1988-1996, 2003.

23. J. Lee, Y. Roh, J. Kim, and H. Kim W. Kim, S. Hong. A Steerable Tangible Interface for Multi-Layered Contents Played on a Tabletop Interface. In DVD of ITS '09. ACM, 2009.

24. J. C. Lee, S. E. Hudson, J. W. Summet, and P. H. Dietz. Moveable Interactive Projected Displays Using Projector Based Tracking. In Proc. of UIST, pp. 63-72, 2005.

25. J. C. Lee, S. E. Hudson, and E. Tse. Foldable Interactive Displays. In Proc. of UIST, pp. 287-290, 2008.

26. D. Molyneaux and H. Gellersen. Projected Interfaces: Enabling Serendipitous Interaction with Smart Tangible Objects. In Proc. of TEI '09, pp. 385-392, 2009.

27. J. Sanneblad and L. E. Holmquist. Ubiquitous Graphics: Combining Hand-held and Wall-size Displays to Interact with Large Images. In Proc. of AVI, pp. 373 377, 2006.

28. M. Sarkar and M. H. Brown. Graphical Fisheye Views. $C A C M, 37(12): 73-83,1994$.
29. C. Schwesig, I. Poupyrev, and E. Mori. Gummi: A Bendable Computer. In Proc. of CHI, pp. 263-270, 2003.

30. B. Shneiderman. Direct Manipulation: A step beyond programming languages. IEEE Computer, 16(8):461467, 1983.

31. M. Spindler and R. Dachselt. Towards Pen-based Annotation Techniques for Tangible Magic Lenses Above a Tabletop. In DVD of ITS '09. ACM, 2009.

32. M. Spindler, S. Stellmach, and R. Dachselt. PaperLens: Advanced Magic Lens Interaction Above the Tabletop. In Proc. of ITS '09, pp. 77-84. ACM, 2009.

33. S. Subramanian, D. Aliakseyeu, and A. Lucero. MultiLayer Interaction for Digital Tables. In Proc. of UIST, pp. 269-272, 2006.

34. C. Thiede, G. Fuchs, and H. Schumann. Smart Lenses. In Proc. of Smart Graphics, pp. 178-189, 2008.

35. C. Tominski, J. Abello, and H. Schumann. Two Novel Techniques for Interactive Navigation of Graph Layouts. Poster at EuroVis '09, 2009.

36. C. Tominski, J. Abello, F. van Ham, and H. Schumann. Fisheye Tree Views and Lenses for Graph Visualization. In Proc. of IEEE InfoVis, pp. 17-24, 2006.

37. C. Tominski, G. Fuchs, and H. Schumann. Task-Driven Color Coding. In Proc. of IEEE InfoVis, pp. 373-380, 2008.

38. B. Ullmer and H. Ishii. The metaDESK: Models and Prototypes for Tangible User Interfaces. In Proc. of UIST, pp. 223-232, 1997.

39. B. Ullmer, H. Ishii, and R. J. K. Jacob. Tangible Query Interfaces: Physically Constrained Tokens for Manipulating Database Queries. In Proc. of Interact '03, pp. 279-286, 2003.

40. F. van Ham and J. J. van Wijk. Interactive Visualization of Small World Graphs. In Proc. of IEEE InfoVis, pp. 199-206, 2004.

41. J. J. van Wijk and W. A. A. Nuij. A Model for Smooth Viewing and Navigation of Large 2D Information Spaces. IEEE TVCG, 10(4):447-458, 2004.

42. S. Voida, M. Tobiasz, J. Stromer, P. Isenberg, and S. Carpendale. Getting Practical with Interactive Tabletop Displays: Designing for Dense Data, "Fat Fingers", Diverse Interactions, and Face-to-Face Collaboration. In Proc. of ITS '09, pp. 109-116, 2009.

43. M. Weiss, J. Wagner, Y. Jansen, R. Jennings, R. Khoshabeh, J. D. Hollan, and J. Borchers. SLAP Widgets: Bridging the Gap Between Virtual and Physical Controls on Tabletops. In Proc. of CHI, pp. 481490, 2009

44. S. White, D. Feng, and S. Feiner. Interaction and Presentation Techniques for Shake Menus in Tangible AR. In Proc. of ISMAR '09, pp. 39-48, 2009.

45. K.-P. Yee. Peephole Displays: Pen Interaction on Spatially Aware Handheld Computers. In Proc. of CHI, pp. $1-8,2003$.

46. J. S. Yi, Y. ah Kang, J.T. Stasko, and J.A. Jacko. Toward a Deeper Understanding of the Role of Interaction in Information Visualization. IEEE TVCG, 13(6):12241231, 2007. 